Recent Results of Meter-decameter Wave Observations of Solar Flares

N. Gopalswamy and M. R. Kundu

Astronomy Program, University of Maryland, College Park, Mn 20742

Abstract

We present recent results from meter-decameter imaging of several classes of solar radio bursts: Preflare activity in the form of type III bursts, correlated type IIIs from distant sources, and type II and moving type IV bursts associated with flares and CMEs.

\title{
1. Intraduction
}

Most of the meter-decameter'radio bursts are signatures of distrubances suq as electron bears, shock waves and plasmoids moving through the solar coror 1. Type III bursts are prompt indicators of acceleration of 10-100 keV electrons. Type II bursts indicate in situ particle acceleration in shock waves during many flares and coronal mass ejections (CMEs). Moving type IV bursts are due to nonthermal electrons trapped in moving magnetic structures in the corona. As temporal association of many of these bursts could lead to $\mathrm{m}$ : leading conclusions, positional information alone can shed light on the exact physical relation between these disturbances. In this paper., we review some recent results from the Clark Lake multifrequency radiohellograph which contributed to the understanding of these phenomena.

\section{Precurser Type IIIs.}

Type III enission in the preflare stage could be a slgnature of preflare electron acceleration (e.g., Kundu, 1986; Kane and Pick, 1976). A comparison of the location of these preflare type IIIs with those of impulsive phase could tell us about the changes in the magnetic fleld structure between the preflare and impulsive phases.

Fig. 1A shows the type III bursts during February 3, 1986 flare in a) preflare, b) preflash, c) Impulsive (early), d) Impulsive (late) and e) decay phases, showing good temporal correlation with the impulsive bursts in hard $X-$ rays and microwaves. As seen in Fig. 1B the source positions of the preflare and impulsive phase type III bursts appear very close, but there is a definite displacement of the source position towards the flare site. The movement of the acceleration region or its expansion due to resistivity increase in the 1mpulsive phase or both might have caused this shift. The source.displacement is larger at lower frequencies suggesting the presence of diverging magnetic fleld lines from the flare site. Eninanced soft X-ray emission was also detected during the preflash type III bursts, implying a possible link between particle acceleration and plasma heating (Kundu et al., 1988). The number of elctrons involved in producing a type III burst and the average electron energy are obtained using the measured characteristics of the radio bursts as $\sim 3-5 \times 10^{33}$ and $10-25 \mathrm{keV}$ respectively (Gopa? swamy and Kundu, 1987).

A type IV burst and a flare behind the $11 \mathrm{mb}$ were assoclated with the 1984, June 27 CME with precursors in soft $X$-rays, Hu, and radio. The flare occurred $\sim 25 \mathrm{~min}$ after the CME onset and about $7 \mathrm{~min}$ after the start of 


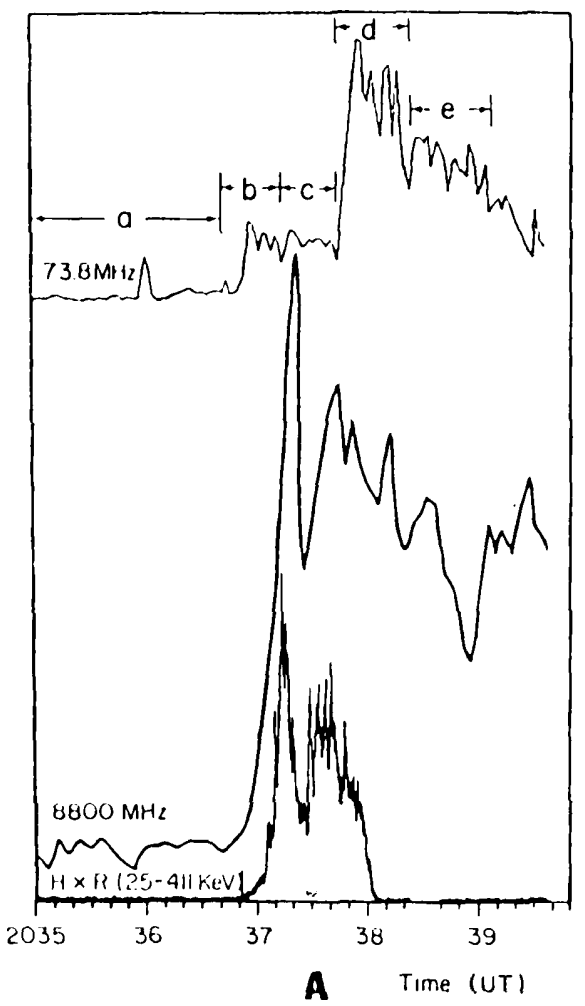

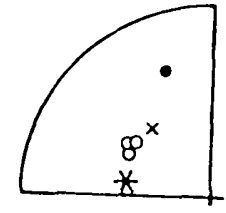

a) Preflare

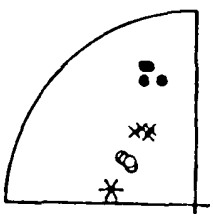

c) Impulsive (early)

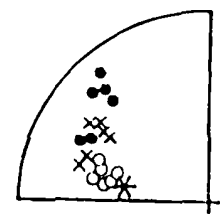

e) Decay

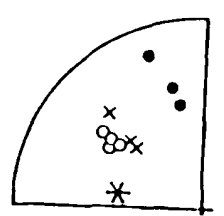

b) Preflash

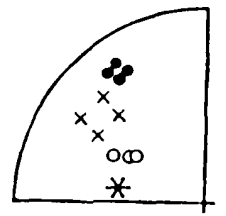

d) Impulsive (late)

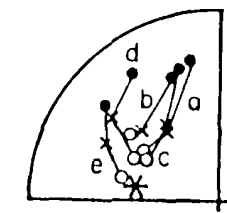

f) Average

B

B) Type III centroids

Fig. 1A: Type III, microwave and hard X-ray bursts; at different phases.

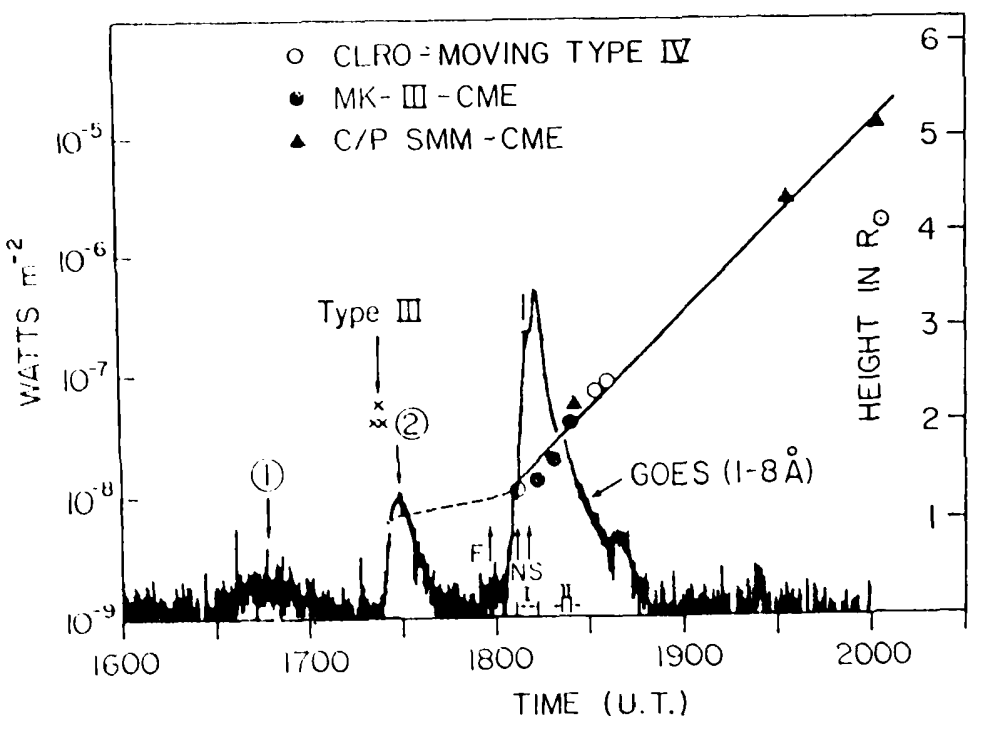

Fig. 2: Type III and soft $X$-ray ( 1 and 2) precursors with CME height time plot. $F, S, N$ denote the start of the filament activity and southern and northern sprays respectively. I and II denote periods of impulsive flare and type II burst. 
prominence motion. The presence of type III bursts and soft $X$-rays prior to the flare onset indicate the presence of particle acceleration and heating in the inner corona (Fig. 2). The observation of type III precursors is significant in that, the soft $X$-ray precursor itself could have been caused by the heating due to accelerated particles. In this case also, the precursor type IIIs were weak compared to impulsive phase ones and they occurred in the same general location in both phases. The number of electrons responsible for producing type III bursts, 1 s $\sim 10^{32}$ (Gopalswamy and Kundu, 1988).

\section{Correlated type IIIs}

Figure $3(a)$ shows the varlation of brightness temperature with time of three type III bursts A, B and C. During each of these peaks, the radiohelfograph 1mages show three sources, one towards north (N) and two towards west (W1 and W2) having simflar time profiles (F1g. 3(b)). It is important to note that emission occurs sinultaneously at locations which are $\sim 10^{6} \mathrm{~km}$ apart. Both $\mathrm{N}$ and $\mathrm{Wl}$ are of the same intensity at $38.5 \mathrm{Mllz}$ and at $50 \mathrm{Mllz}, \mathrm{Wl}$ stronger than $N$ during the peak $B$ and the opposite is true during the next peak (C). There is no delay between $N$ and $H 1$ which is expected to be $24 \mathrm{~s}$ if
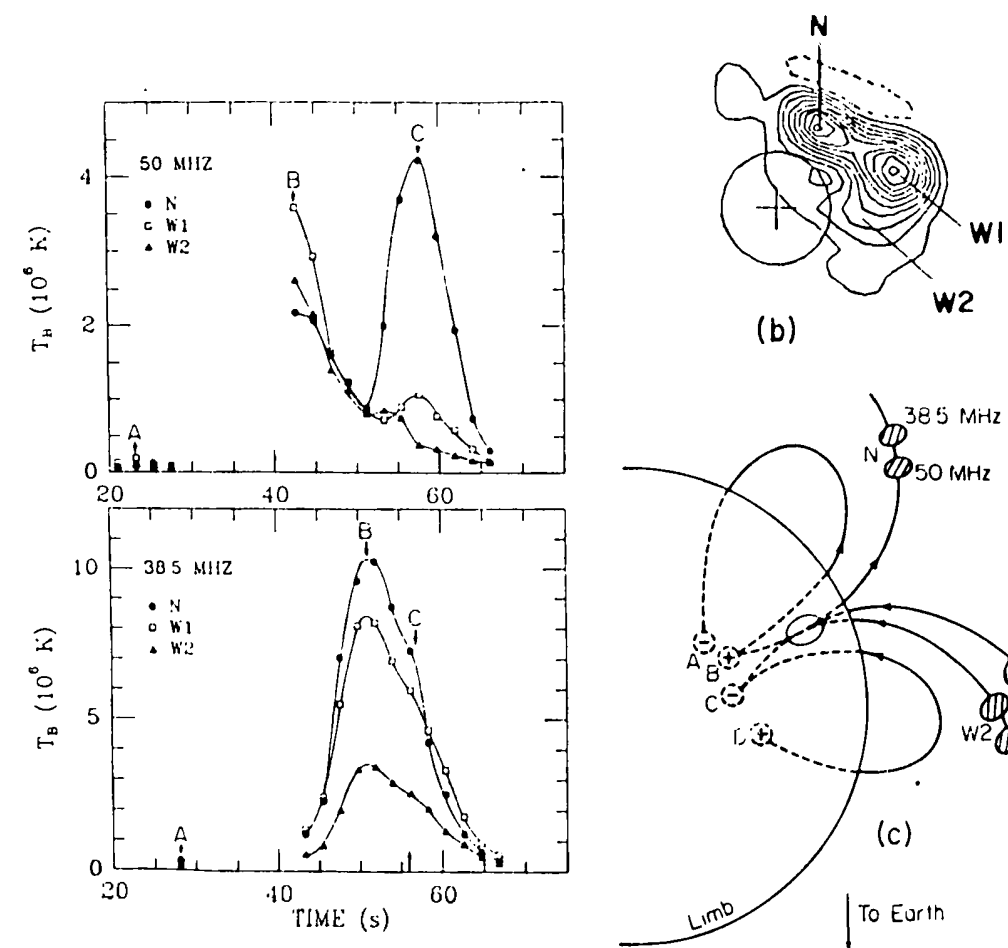

(b)

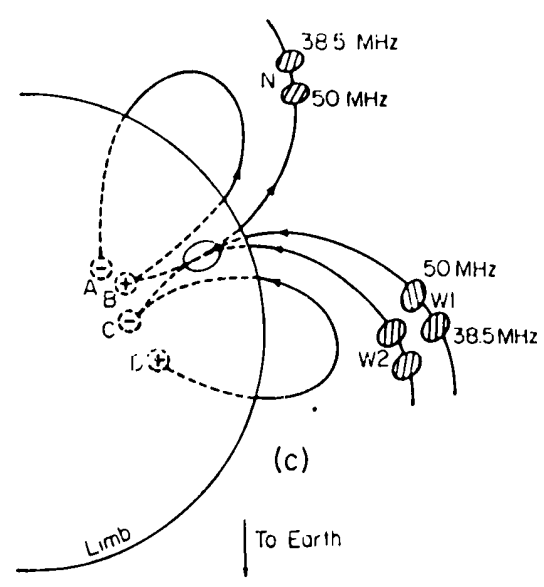

(a)

Fig. 3: a) Brightness temperature of type III bursts; b) location of $\mathrm{N}$, Wl, W2 sources; c) model of source region. 
they constitute a direct and reflected source pair. The observed drift rates do not suggest a U-burst type of magnetic field. The possibile structure of mafnetic field lines emanating from the acceleration region is shown in Fig. 3c. The angiular extent covered by these field lines must be very large, $26^{\circ}$, in order that the electrons propagating along these lines produce the observed correlated sources (Kunciu and Copalswamy, 1987).

\section{Relation Among CME, Type II, Type IV}

It 1 s presently belleved that flare is a secondary process in a CME event and the lift-off of the latter is supposed to precede the flare by a few mlnutes (see e.g. Wagner, 1984). A flare blast wave can produce type II bursts. A super Alfvenic CME can also produce a shock ahead of it. This demands a spectfic spatial relation between CME or moving type IV and type II - the type II must be at or ahead of the CMF and move with nearly same speed. Sfmultaneous observation's show that the type II location could be ahead of CAS because its higher speed and also can have different location with respect to CME or moving type IV.

On February 17, the Sill $\mathrm{C} / \mathrm{P}$ coronagraph observed a streamer disruption event on the east $1 \mathrm{lmb}$. The Clark Lake radiohellograph detected the onset of a type II burst at the NE limb at 20:25 UT (F1gure 4(a), marked A) followed by a moving, source (B) after $10 \mathrm{~min}$ below the rype $\mathrm{II}$, with a sped of $\sim 200 \mathrm{~km}$ $\mathrm{s}^{-1}$. Fig. $4(\mathrm{~b})$ shows that the type II burst (A) has a parallel to limb motion

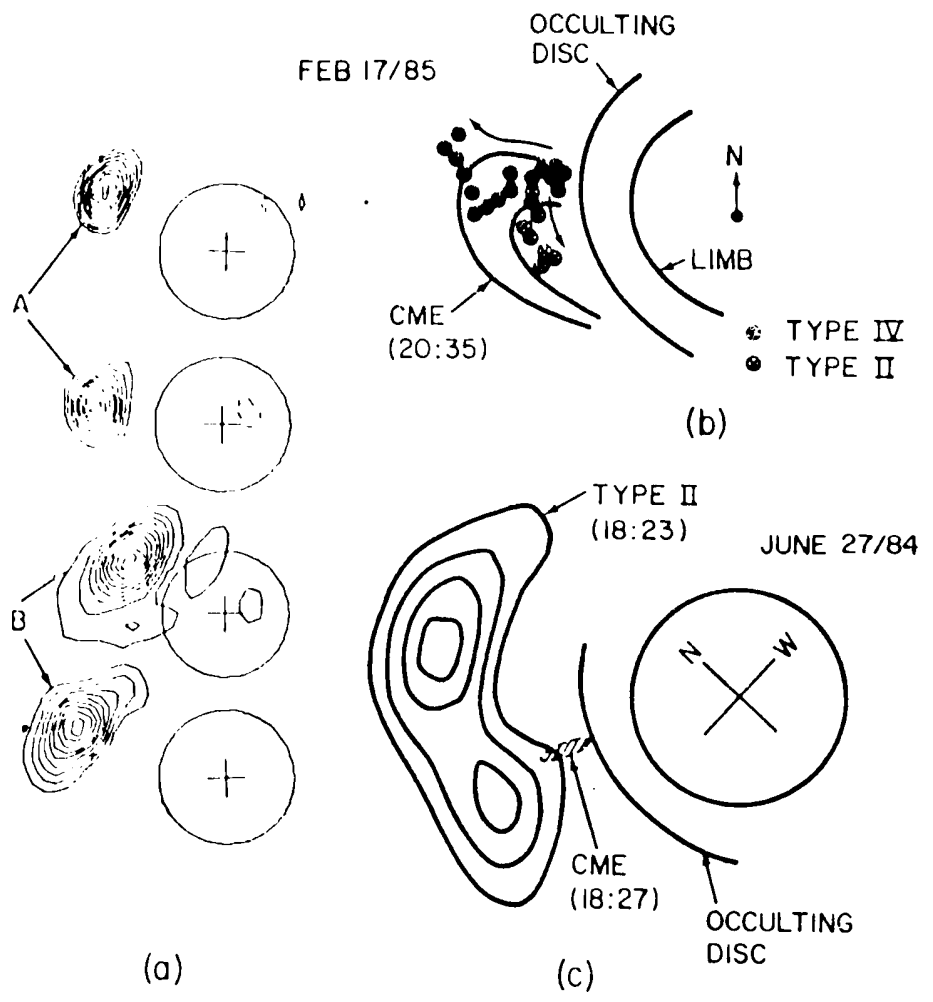

F1g. 4: a) Type II (A) and moving type IV (B) bursts; b) their centroids of February 17, 1985 event; C) type II and CME of June 27, 1984. 
and moving type IV has a nearly radial motion. There was no significant surface activity reported in association with this event. A small GOES soft $X$-ray flare (1mportance BI.2) occuried at 20:06 UI', and may well indicate the start of the event. If we assume a blast wave infliated at the time of the COES flare, the lmplied velocity needed to reach $50 \mathrm{MIlz}$ level at the observed onset time is $1000 \mathrm{~km} / \mathrm{s}$, which is the normal type II speed. From parallel to limb motion of the type II centrold one can estimate a speed of $\sim 1300 \mathrm{~km} / \mathrm{s}$, clearly much higher than the moving type IV speed. The type II and moving type IV do not seem to have a physical relation as one might expect in a piston driven case. Therefore, both positional analysis and speed show that the type II is created by a decoupled shock. The energetic particles responsible for type IV might have come from either reconnection during liftoff of the CME or due to the passage of type II shock through the CME.

Figure 2 provides a summary of the time history of the June 27, 1984 CME as observed in both radio and white light. The CME appeared as a single clump of material moving out with a speed of $\sim 350 \mathrm{~km} \mathrm{~s}^{-1}$. Both type IV radio sources and white light CMF follow the same direction and are co-spatial. The type II burst observed at 18:22 UT has a large extended structure with two prominent centroids, one ahead of the CME and the other far north of 1 . The centroids are separated by a distance of $\sim 2.2 \mathrm{R}_{0}$. The overall size of the type II source is $\sim 20$ times bigger than the visible ejection. Fig. $4 \mathrm{c}$ shows that the nearest centroid type II at 18:23 UT is $\sim .4 \mathrm{R}_{0}$ ahead of the CME leading edge at 18:27 UT and the farthest one was $\sim 1.5 \mathrm{R}_{0}$ away. Clearly the CME could not have generated such a huge shock. On the other hand, if the shock were generated durfing the impulsive phase of the flare, then 1 t takes $9 \mathrm{~min}$ to reach the observed height implying a speed of $\sim 1500 \mathrm{~km} \mathrm{~s}^{-1}$. This is $\sim 5$ times larger than the speed of the CME (and type IV) and hence might have overtaken the CME.

\section{References}

Gopalswamy, N., and Kundu, M.R.: 1987, Solar Phys. 111, 347. Gopalswamy, N., and Kundu, M.R.: 1988, Solar Phys. 114, 347. Kane, S.R. and Pick, M.: 1976, Solar Phys. 47, 293.

Kundu, M.R.: 1986, Adv. Space Res. 6, 93.

Kundu, M.R. and Gopalswamy, N.: 1987, Solar Phys. 112, 133.

Kundu, M.R. et a1.: 1988, Solar Phys. 114, 273.

Wagner, W.J.: 1984, Ann. Rev. Astron. Astrophys. 22, 267. 